\title{
IRREDUCIBLE HARISH CHANDRA MODULES OVER THE DERIVATION ALGEBRAS OF RATIONAL QUANTUM TORI
}

\author{
GENQIANG LIU \\ College of Mathematics and Information Science, Henan University, Kaifeng 475004, China \\ e-mail: liugenqiang@amss.ac.cn \\ and KAIMING ZHAO \\ Department of Mathematics, Wilfrid Laurier University, Waterloo, ON N2L 3C5, \\ Canada, and College of Mathematics and Information Science, \\ Hebei Normal (Teachers) University, Shijiazhuang, Hebei 050016, China \\ e-mail:kzhao@wlu.ca
}

(Received 22 July 2012; accepted 1 August 2012; first published online 25 February 2013)

\begin{abstract}
Let $d$ be a positive integer, $q=\left(q_{i j}\right)_{d \times d}$ be a $d \times d$ matrix, $\mathbb{C}_{q}$ be the quantum torus algebra associated with $q$. We have the semidirect product Lie algebra $\mathfrak{g}=\operatorname{Der}\left(\mathbb{C}_{q}\right) \ltimes Z\left(\mathbb{C}_{q}\right)$, where $Z\left(\mathbb{C}_{q}\right)$ is the centre of the rational quantum torus algebra $\mathbb{C}_{q}$. In this paper, we construct a class of irreducible weight $\mathfrak{g}$-modules $\mathcal{V}^{\alpha}$ $(V, W)$ with three parameters: a vector $\alpha \in \mathbb{C}^{d}$, an irreducible $\mathfrak{g l}_{d}$-module $V$ and a graded-irreducible $\mathfrak{g l}_{N}$-module $W$. Then, we show that an irreducible Harish Chandra (uniformaly bounded) $\mathfrak{g}$-module $M$ is isomorphic to $\mathcal{V}^{\alpha}(V, W)$ for suitable $\alpha, V, W$, if the action of $Z\left(\mathbb{C}_{q}\right)$ on $M$ is associative (respectively nonzero).
\end{abstract}

2000 Mathematics Subject Classification. 17B10, 17B20, 17B65, 17B66, 17B68.

1. Introduction. The classification of simple weight modules is a classical problem in the representation theory of Lie algebras. Simple weight modules with finitedimensional weight spaces (also called Harish Chandra modules) are classified for several classes of algebras, including simple finite-dimensional Lie algebras [26] and various generalized Virasoro algebras [24, 25, 27]. For complete classification of irreducible weight modules (not necessarily Harish Chandra modules), however, it is solved only for the Lie algebra $\mathfrak{s l}_{2}$ (P. Gabriel, Lectures at the Seminaire Godement, Paris, unpublished notes, 1959; see [28, chapter 3] for details).

Let $q=\left(q_{i j}\right)_{d \times d}$ be a $d \times d$ complex matrix satisfying $q_{i i}=1, q_{i j}=q_{j i}^{-1}$ with all $q_{i j}$ being roots of unity. In this paper, we consider the rational quantum torus algebra $\mathbb{C}_{q}$ associated with $q$, and its derivation algebra $\operatorname{Der}\left(\mathbb{C}_{q}\right)$. The algebra $\mathbb{C}_{q}$ is an important algebra, since it is the coordinate algebra of a large class of extended affine Lie algebras (see [3]) and shows up in the theory of non-commutative geometry (see [13]). When all $q_{i j}=1$, the algebra $\operatorname{Der}\left(\mathbb{C}_{q}\right)$ is the classical Witt algebra $W_{d}$, i.e. the derivation algebra of the Laurent polynomial algebra $A=\mathbb{C}\left[x_{1}^{ \pm 1}, x_{2}^{ \pm 1}, \ldots, x_{d}^{ \pm 1}\right]$ (see [31]). In particular, when $d=1, \operatorname{Der}\left(\mathbb{C}_{q}\right)$ is the centreless Virasoro algebra which has been widely used in many mathematics and physics branches (see [17]).

Modules over Witt algebra are used in the classification of irreducible Harish Chandra modules over finite-dimensional Lie algebras [26], also closely connected 
with extended affine Lie algebra theory [1], toroidal Lie algebras $[\mathbf{4}, \mathbf{1 1}, \mathbf{1 2}]$ and physics [19-23].

In recent years, representations for Witt algebras have attracted much attention from many mathematicians and physicists (see [7, 9, 10, 14, 15, 18-22, 29], and [33]). The result in [29] asserted that any irreducible Harish Chandra $W_{d}$-module is either dense (with uniformly bounded weight spaces) or punctured (also with uniformly bounded weight spaces) or is an irreducible quotient of some generalized Verma module induced from the previous two classes of modules. Some of the latter classes of modules are constructed in [8] (hopefully all), which depend completely on the first two classes of weight modules. Some dense and punctured modules were introduced and studied in $[9,32]$, which are called Shen's modules or Larsson's modules. It is generally considered that Shen's modules exhaust dense or punctured modules over $W_{d}$ (see $\left.[\mathbf{1 0}, \mathbf{1 5}]\right)$.

Irreducible Harish Chandra modules over the semidirect product Lie algebra $\operatorname{Der}\left(\mathbb{C}_{q}\right) \ltimes \mathbb{C}_{q}$ with all $q_{i j}=1$ were classified in [10] and [14]. This paper generalizes these results to the semidirect product Lie algebra $\operatorname{Der}\left(\mathbb{C}_{q}\right) \ltimes Z\left(\mathbb{C}_{q}\right)$ for rational quantum torus $\mathbb{C}_{q}$, where $Z\left(\mathbb{C}_{q}\right)$ is the centre of $\mathbb{C}_{q}$.

This paper is organized as follows. In Section 2, we recall some known Lie algebras and results for later use. In particular, the Lie algebra $\mathfrak{g}=W_{d} \ltimes \mathbb{C}_{q}$ is isomorphic to $\operatorname{Der}\left(\mathbb{C}_{q}\right) \ltimes Z\left(\mathbb{C}_{q}\right)$. In Section 3 , we construct $\mathbb{Z}^{d}$-graded modules $\mathcal{V}(W)$ over the Lie algebra $\mathbb{C}_{q}$ for any $\Gamma$-graded $\mathfrak{g l}_{N}$-module $W$ (not necessarily finite dimensional), where $\Gamma$ is defined in Section 2.2 and $N$ is defined in Section 3.1, and show that they are all $\mathbb{Z}^{d}$-graded modules over the Lie algebra $\mathbb{C}_{q}$ with associative action of the centre $Z\left(\mathbb{C}_{q}\right)$. In Section 4, we construct irreducible Harish Chandra $\mathfrak{g}$-modules $\mathcal{V}^{\alpha}(V, W)$ and $F^{\alpha}(V)$ for any irreducible $\mathfrak{g l}_{d}$-module $V$, graded-irreducible $\mathfrak{g l}_{N}$-module $W=\bigoplus_{\bar{n} \in \Gamma} W_{\bar{n}}$, and $\alpha \in \mathbb{C}^{d}$. We show that if the action of $Z\left(\mathbb{C}_{q}\right)$ is associative on an irreducible Harish Chandra $\mathfrak{g}$-module $M$, then $M$ has to be isomorphic to $\mathcal{V}^{\alpha}(V, W)$ for suitable $\alpha, V, W$ (Theorem 4.4). We also prove that any uniformly bounded irreducible $\mathfrak{g}$-module with the nonzero action of $Z\left(\mathbb{C}_{q}\right)$ has to be isomorphic to $\mathcal{V}^{\alpha}(V, W)$ for suitable $\alpha, V, W$ (Theorem 4.6). We use the main results of [10] and the density theorem from ring theory. The technique in the main proof of this paper is to construct a finite-dimensional representation for a solvable subalgebra so that the Lie theorem can be applied.

\section{Notation and preliminaries.}

2.1. Witt algebras $W_{d}$. We denote by $\mathbb{Z}, \mathbb{Z}_{+}, \mathbb{N}, \mathbb{Q}$ and $\mathbb{C}$ the sets of all integers, nonnegative integers, positive integers, rational numbers and complex numbers, respectively. For a Lie algebra $\mathfrak{g}$, we denote its universal enveloping algebra by $U(\mathfrak{g})$.

We fix a positive integer $d>1$ and denote by $W_{d}$ the derivation Lie algebra of the Laurent polynomial algebra $A=\mathbb{C}\left[x_{1}^{ \pm 1}, x_{2}^{ \pm 1}, \ldots, x_{d}^{ \pm 1}\right]$. For $i \in\{1,2, \ldots, d\}$, set $\partial_{i}=x_{i} \frac{\partial}{\partial x_{i}}$; and for any $a=\left(a_{1}, a_{2}, \ldots, a_{d}\right)^{T} \in \mathbb{Z}^{d}$ (considered as column vectors), set $x^{a}=x_{1}^{a_{1}} x_{2}^{a_{2}} \cdots x_{d}^{a_{d}}$.

We fix the vector space $\mathbb{C}^{d}$ of $d \times 1$ matrices. Denote the standard basis by $\left\{e_{1}, e_{2}, \ldots, e_{d}\right\}$. Let $(\cdot \mid \cdot)$ be the standard symmetric bilinear form such that $(u \mid v)=$ $u^{T} v \in \mathbb{C}$, where $u^{T}$ is the matrix transpose of $u$. For $u \in \mathbb{C}^{d}$ and $r \in \mathbb{Z}^{d}$, we denote $D^{\prime}(u, r)=x^{r} \sum_{i=1}^{d} u_{i} \partial_{i} \in W_{d}$. Then we have

$$
\left[D^{\prime}(u, r), D^{\prime}(v, s)\right]=D^{\prime}(w, r+s), u, v \in \mathbb{C}^{d}, r, s \in \mathbb{Z}^{d},
$$


where $w=(u \mid s) v-(v \mid r) u$. Note that for any $u, v, z, y \in \mathbb{C}^{d}$, both $u v^{T}$ and $x y^{T}$ are $d \times d$ matrices, and $\left(u v^{T}\right)\left(z y^{T}\right)=(v \mid z) u y^{T}$.

2.2. Rational quantum torus. Let $q=\left(q_{i j}\right)_{i, j=1}^{d}$ be a $d \times d$ matrix over $\mathbb{C}$ satisfying $q_{i i}=1, q_{i j}=q_{j i}^{-1}$, where $q_{i j}$ are roots of unity for all $1 \leqslant i, j \leqslant d$. We will call such a matrix $q$ rational. The rational quantum torus $\mathbb{C}_{q}$ (see [30]) is the unital associative algebra over $\mathbb{C}$ generated by $t_{1}^{ \pm 1}, \ldots, t_{d}^{ \pm 1}$ and subject to the defining relations $t_{i} t_{j}=$ $q_{\ddot{j}} t_{j} t_{i}, t_{i} t_{i}^{-1}=t_{i}^{-1} t_{i}=1$ for all $1 \leqslant i, j \leqslant d$.

Similarly, $t^{n}=t_{1}^{n_{1}} t_{2}^{t_{2}} \cdots t_{d}^{n_{d}}$ for any $n=\left(n_{1}, \ldots, n_{d}\right)^{T} \in \mathbb{Z}^{d}$.

For any $n, m \in \mathbb{Z}^{d}$, we define the functions $\sigma(n, m)$ and $f(n, m)$ by

$$
t^{n} t^{m}=\sigma(n, m) t^{n+m}, t^{n} t^{m}=f(n, m) t^{m} t^{n} .
$$

It is well known that

$$
\sigma(n, m)=\prod_{1 \leq i<j \leq d} q_{j i}^{n_{j} m_{i}}, f(n, m)=\prod_{i, j=1}^{d} q_{j i}^{n_{j} m_{i}},
$$

and $f(n, m)=\sigma(n, m) \sigma(m, n)^{-1}$. We also define

$$
\operatorname{rad}(f)=\left\{n \in \mathbb{Z}^{d} \mid f\left(n, \mathbb{Z}^{d}\right)=1\right\}, \Gamma=\mathbb{Z}^{d} / \operatorname{rad}(f) .
$$

Since $\mathbb{C}_{q}$ is an associative algebra, it is naturally a Lie algebra defined by the usual brackets. For each $n \in \mathbb{Z}^{d}$, we denote its image in $\Gamma$ by $\bar{n}$. Then, $\mathbb{C}_{q}$ is a $\Gamma$-graded Lie algebra with the decomposition $\mathbb{C}_{q}=\bigoplus_{\bar{n} \in \Gamma}\left(\mathbb{C}_{q}\right)_{\bar{n}}$, where $\left(\mathbb{C}_{q}\right)_{\bar{n}}=\sum_{r \in \operatorname{rad}(f)} \mathbb{C} t^{n+r}$.

From the results in [30], we have the following lemma.

LEMMA 2.1. Up to an isomorphism of $\mathbb{C}_{q}$, we may assume that $q_{2 i, 2 i-1}=q_{i}, q_{2 i-1,2 i}=$ $q_{i}^{-1}$, for $1 \leqslant i \leqslant z$, and other entries of $q$ are all 1 , where $z \in \mathbb{N}$ with $2 z \leqslant d$ and the orders $k_{i}$ of $q_{i}, 1 \leqslant i \leqslant z$ as roots of unity satisfy $k_{i+1} \mid k_{i}, 1 \leqslant i<z$. Then

$$
\operatorname{rad}(f)=\bigoplus_{i=1}^{z}\left(k_{i} \mathbb{Z} e_{2 i-1} \oplus k_{i} \mathbb{Z} e_{2 i}\right) \oplus \bigoplus_{j=2 z+1}^{d} \mathbb{Z} e_{j} .
$$

If $q$ has the form in Lemma 2.1, then $\sigma(r, n)=1$ (i.e. $t^{n} t^{r}=t^{n+r}$ ) for all $r \in \operatorname{rad}(f)$ and $n \in \mathbb{Z}^{d}$. This will be used many times later.

2.3. The bigger derivation algebra of the rational quantum torus $\mathbb{C}_{q}$. Let $\mathfrak{g}=$ $\operatorname{Der}\left(\mathbb{C}_{q}\right) \ltimes Z\left(\mathbb{C}_{q}\right)$ be the semidirect product Lie algebra, where $Z\left(\mathbb{C}_{q}\right)=\oplus_{r \in \operatorname{rad}(f)} \mathbb{C} t^{r}$ is the centre of $\mathbb{C}_{q}$. Note that $\operatorname{Der}\left(\mathbb{C}_{q}\right)$ acts on $Z\left(\mathbb{C}_{q}\right)$ as derivations. Let $\mathfrak{g}_{n}$ be the set of homogeneous elements of $\mathfrak{g}$ with degree $n$. Then, from Lemma 2.48 of [3], we have

$$
\mathfrak{g}=\bigoplus_{n \in \mathbb{Z}^{d}} \mathfrak{g}_{n}, \mathfrak{g}_{n}= \begin{cases}\mathbb{C a d}\left(t^{n}\right), & \text { if } n \notin \operatorname{rad}(f), \\ \mathbb{C} t^{n} \bigoplus_{i=1}^{d} \mathbb{C} t^{n} \partial_{i}, & \text { if } n \in \operatorname{rad}(f) .\end{cases}
$$

Recall that $\partial_{i}\left(t^{n}\right)=n_{i} t^{n}$ for any $n \in \mathbb{Z}^{d}$. We will simply denote $\operatorname{ad}\left(t^{n}\right)$ in $\mathfrak{g}$ by $t^{n}$ for $n \notin \operatorname{rad}(f)$. 
For $n \in \operatorname{rad}(f), u \in \mathbb{C}^{d}$, we still denote $D(u, n)=t^{n} \sum_{i=1}^{d} u_{i} \partial_{i}$. Lie brackets of $\mathfrak{g}$ are given by

(1) $\left[t^{s}, t^{s^{\prime}}\right]=\left(\sigma\left(s, s^{\prime}\right)-\sigma\left(s^{\prime}, s\right)\right) t^{s+s^{\prime}}, \forall s, s^{\prime} \in \mathbb{Z}^{d}$;

(2) $\left[D(u, r), t^{s}\right]=(u \mid s) \sigma(r, s) t^{r+s}, \forall s \in \mathbb{Z}^{d}, r \in \operatorname{rad}(f), u \in \mathbb{C}^{d}$;

(3) $\left[D(u, r), D\left(u^{\prime}, r^{\prime}\right)\right]=D\left(w, r+r^{\prime}\right), \forall r, r^{\prime} \in \operatorname{rad}(f), u, u^{\prime} \in \mathbb{C}^{d}$, where $w=\sigma\left(r, r^{\prime}\right)\left(\left(u \mid r^{\prime}\right) u^{\prime}-\left(u^{\prime} \mid r\right) u\right)$.

We can see that $\mathfrak{g}_{0}=\operatorname{span}\left\{t^{0}, D(u, 0) \mid u \in \mathbb{C}^{d}\right\}$ is the Cartan subalgebra of $\mathfrak{g}$, and the subalgebra of $\mathfrak{g}$ spanned by $\left\{t^{s} \mid s \in \mathbb{Z}^{d}\right\}$ is isomorphic to the Lie algebra $\mathbb{C}_{q}$ which will be identified with $\mathbb{C}_{q}$. Let us also denote by $W_{d}$ the subalgebra $\operatorname{span}\{D(u, r) \mid$ $\left.r \in \operatorname{rad}(f), u \in \mathbb{C}^{d}\right\}$, which is indeed isomorphic to the corresponding Witt algebra (see Lemma 2.3). Thus, $\mathfrak{g}=W_{d} \ltimes \mathbb{C}_{q}$. For convenience, we denote the subalgebra $W_{d} \ltimes Z\left(\mathbb{C}_{q}\right)$ of $\mathfrak{g}$ by $\dot{\mathfrak{g}}$.

A $\mathfrak{g}$-module $V$ is called a weight module provided that the action of $\mathfrak{g}_{0}$ on $V$ is diagonalizable. For any weight module $V$, we have the weight space decomposition

$$
V=\bigoplus_{\lambda \in \mathfrak{g}_{0}^{*}} V_{\lambda},
$$

where $\mathfrak{g}_{0}^{*}=\operatorname{Hom}_{\mathbb{C}}\left(\mathfrak{g}_{0}, \mathbb{C}\right)$ and

$$
V_{\lambda}=\left\{v \in V \mid \partial v=\lambda(\partial) v \text { for all } \partial \in \mathfrak{g}_{0}\right\} .
$$

The space $V_{\lambda}$ is called the weight space corresponding to the weight $\lambda$. The support $\operatorname{supp}(V)$ of the weight module $V$ is defined as the set of all weights $\lambda$ for which $V_{\lambda} \neq 0$. If $V$ is a weight $\mathfrak{g}$-module and $\operatorname{dim}_{\mathbb{C}} V_{\lambda}<\infty$ for all $\lambda \in \mathfrak{g}_{0}^{*}$, the module $V$ is called a Harish Chandra module. If there is an integer $k \in \mathbb{N}$ such that $\operatorname{dim}_{\mathbb{C}} V_{\lambda}<k$ for all $\lambda \in \mathfrak{g}_{0}^{*}$, the module $V$ is called a uniformly bounded module.

If $V$ is an irreducible weight module over $\mathfrak{g}$, then the central element $t^{0}$ acts on $V$ as a scalar. In this case, a weight $\lambda$ of $V$ can be determined by $\left(\lambda\left(\partial_{1}\right), \ldots, \lambda\left(\partial_{d}\right)\right)$. Then, we consider weights as elements in $\mathbb{C}^{d}$. For example, $\mathbb{Z}^{d}$ is the weight set of the adjoint representation of $\mathfrak{g}$ (i.e. $\alpha\left(\partial_{i}\right)=\alpha_{i}$ for all $\alpha \in \mathbb{Z}^{d}$ ). Furthermore, if $V$ is a weight $\mathfrak{g}$-module, then for all $\alpha \in \mathbb{Z}^{d}$ and $\lambda \in \mathfrak{g}_{0}^{*}$ we have $\mathfrak{g}_{\alpha} V_{\lambda} \subset V_{\lambda+\alpha}$. From this, it follows that if $V$ is an indecomposable weight module (in particular, simple), then $\operatorname{supp}(V) \subset \lambda+\mathbb{Z}^{d}$ for some $\lambda \in \mathfrak{g}_{0}^{*}$.

2.4. Other preliminary results. The following result is well known. For a proof, see Lemma 2.2 in [23].

LeMmA 2.2. If $\mathbb{C}_{q}^{\prime}=\left[\mathbb{C}_{q}, \mathbb{C}_{q}\right] \neq 0$, then it is a $\mathbb{Z}^{d}$-graded-simple Lie algebra.

Let $L=\operatorname{Der}(A) \ltimes A$, where $A$ is the Laurent polynomial algebra $\mathbb{C}\left[x_{1}^{ \pm 1}\right.$, $\left.x_{2}^{ \pm 1}, \ldots, x_{d}^{ \pm 1}\right]$.

\section{LEMMA 2.3. As Lie algebras, $\dot{\mathfrak{g}} \cong L$.}

Proof. We may assume that $q$ has the form in Lemma 2.1. Then $\operatorname{rad}(f)=$ $\bigoplus_{i=1}^{z}\left(k_{i} \mathbb{Z} e_{2 i-1} \oplus k_{i} \mathbb{Z} e_{2 i}\right) \oplus \bigoplus_{j=2 z+1}^{d+1} \mathbb{Z} e_{j}$. Let

$$
B=\operatorname{diag}\left(k_{1}, k_{1}, k_{2}, k_{2}, \ldots, k_{z}, k_{z}, 1, \ldots, 1\right)
$$


be the $d \times d$ diagonal matrix. Note that $B$ is generally not in $\operatorname{GL}(d, \mathbb{Z})$. Then it is easy to check the linear map

$$
\begin{aligned}
\rho: \dot{\mathfrak{g}} & \rightarrow L, \\
t^{B n} & \mapsto x^{n}, \\
D(u, B n) & \mapsto D^{\prime}\left(B^{T} u, n\right),
\end{aligned}
$$

where $D^{\prime}(u, n)=x^{n} \sum_{i=1}^{d} u_{i} x_{i} \frac{\partial}{\partial x_{i}}, u \in \mathbb{C}^{d}, n \in \mathbb{Z}^{d}$, is a Lie algebra isomorphism.

The following theorem was proved in [14].

THEOREM 2.4. Let $V$ be an irreducible uniformly bounded weight L-module. Let $x^{0} v=c v$ for any $v \in V$, where $c \in \mathbb{C}$. Then, $c \neq 0$ if and only if $A V \neq 0$. Moreover, when $c \neq 0$, we have

$$
x^{m} x^{s} v=c x^{m+s} v
$$

for any $m, s \in \mathbb{Z}^{d}, v \in V$.

Let us recall the Schur's lemma and density theorem in ring theory, which will be used in the following sections.

Lemma 2.5. Let $M=\bigoplus_{g \in G} M_{g}$ be a G-graded-irreducible module over a G-graded associative $\mathbb{C}$-algebra $R$ of countable dimension, where $G$ is an abelian group. Then, $\operatorname{End}_{R}(M)_{0}=\left\{f \in \operatorname{End}_{R}(M) \mid f\left(M_{g}\right) \subseteq M_{g}\right.$ for all $\left.g \in G\right\}=\mathbb{C}$.

Proof. Since $M=\oplus_{g \in G} M_{g}$ is a $G$-graded-simple module over the $G$-graded associative algebra $R, \operatorname{End}_{R}(M)_{0}$ is a division algebra over $\mathbb{C}$. If $\operatorname{End}_{R}(M)_{0} \neq \mathbb{C}$, then it contains a subfield isomorphic to the field $\mathbb{C}(x)$ of rational functions of an indeterminant $x$. On the other hand, $\operatorname{dim}_{\mathbb{C}} \operatorname{End}_{R}(M)_{0} \leqslant \operatorname{dim}_{\mathbb{C}} R$, thus $\operatorname{dim}_{\mathbb{C}} \operatorname{End}_{R}(M)_{0}$ is finite or countable. However, $\operatorname{dim}_{\mathbb{C}} \mathbb{C}(x)$ is uncountable. Hence, $\operatorname{End}_{R}(M)_{0}=\mathbb{C}$.

THEOREM 2.6. (Density theorem)

(1) (See [16], $p$. 197) Let $M$ be a completely reducible module over a ring $R$. Let $R^{\prime}=\operatorname{End}_{R}(M), R^{\prime \prime}=\operatorname{End}_{R^{\prime}}(M)$. Let $\left\{w_{i} \mid i \in I\right\}$ be a finite subset of $M, a^{\prime \prime}$ an element of $R^{\prime \prime}$. Then there exists an $a \in R$ such that $a w_{i}=a^{\prime \prime} w_{i}$, for all $i \in I$.

(2) (See Theorem A.1 in [6]) Let $M=\oplus_{g \in G} M_{g}$ be a G-graded-completely reducible module over a $G$-graded associative $\mathbb{C}$-algebra $R$, where $G$ is an abelian group. Let $R^{\prime}=\operatorname{End}_{R}(M)_{0}, R^{\prime \prime}=\operatorname{End}_{R^{\prime}}(M)_{0}$. Let $\left\{w_{i} \mid i \in I\right\}$ be a finite subset of $M_{g}$ for some $g \in G, a^{\prime \prime}$ an element of $R^{\prime \prime}$. Then there exists an $a \in R$ such that a $w_{i}=a^{\prime \prime} w_{i}$, for all $i \in I$.

3. $\mathbb{Z}^{d}$-graded modules over the Lie algebra $\mathbb{C}_{q}$. In this section, we assume that $q$ is of the form as Lemma 2.1. In this case, we know that $\left.\Gamma=\oplus_{i=1}^{z}\left(\mathbb{Z} /\left(k_{i} \mathbb{Z}\right)\right) \oplus\left(\mathbb{Z} /\left(k_{i} \mathbb{Z}\right)\right)\right)$.

3.1. A realization of $\mathbb{C}_{q}$. Let $\mathcal{I}=\operatorname{span}\left\{t^{n+r}-t^{n} \mid n \in \mathbb{Z}^{d}, r \in \operatorname{rad}(f)\right\}$, which is an ideal of the associative algebra $\mathbb{C}_{q}$. Then from [30] and [34], we know that $\mathbb{C}_{q} / \mathcal{I} \simeq$ $\otimes_{i=1}^{z} \mathfrak{g l}_{k_{i}} \simeq \mathfrak{g l}_{N}$ as associative algebras with $N=\prod_{i=1}^{z} k_{i}$. It is well known that $\mathfrak{g l}_{k_{i}}$, 
$1 \leqslant i \leqslant z$, as the associative algebra $M_{k_{i}}(\mathbb{C})$, is generated by $X_{2 i-1}, X_{2 i}$ with

$$
\begin{aligned}
& X_{2 i-1}=E_{1,1}+q_{i} E_{2,2}+\cdots+q_{i}^{k_{i}-1} E_{k_{i}, k_{i}}, \\
& X_{2 i}=E_{1,2}+E_{2,3}+\cdots+E_{k_{i}-1, k_{i}}+E_{k_{i}, 1},
\end{aligned}
$$

which satisfy $X_{2 i}^{k_{i}}=X_{2 i-1}^{k_{i}}=1, X_{2 i} X_{2 i-1}=q_{i} X_{2 i-1} X_{2 i}$. We denote $\otimes_{i=1}^{z} X_{2 i-1}^{n_{2 i-1}} X_{2 i}^{n_{2 i}}$ by $X^{n}$ for each $n \in \mathbb{Z}^{d}$. Identifying $\mathfrak{g l}_{N}$ with $\bigotimes_{i=1}^{z} \mathfrak{g l}_{k_{i}}$ as associative algebras, $\mathfrak{g l}_{N}$ is spanned by $X^{n}, n \in \mathbb{Z}^{d}$ and $X^{r}$ equals the identity matrix $E$ in $\mathfrak{g l}_{N}$ for each $r \in \operatorname{rad}(f)$.

LEMMA 3.1. (see [2]) As associative algebras,

$$
\mathbb{C}_{q} \cong \bigoplus_{n \in \mathbb{Z}^{d}}\left(\mathbb{C} X^{n} \otimes x^{n}\right)
$$

where the right-hand side is a $\mathbb{Z}^{d}$-graded subalgebra of the associative algebra $\mathfrak{g l}_{N} \otimes$ $\mathbb{C}\left[x_{1}^{ \pm 1}, x_{2}^{ \pm 1}, \ldots, x_{d}^{ \pm 1}\right]$ and $\mathbb{C}\left[x_{1}^{ \pm 1}, x_{2}^{ \pm 1}, \ldots, x_{d}^{ \pm 1}\right]$ is the Laurent polynomial algebra.

Proof. It is easy to see that the linear map

$$
\begin{aligned}
\varphi: \mathbb{C}_{q} & \rightarrow \bigoplus_{n \in \mathbb{Z}^{d}}\left(\mathbb{C} X^{n} \otimes x^{n}\right), \\
t^{n} & \mapsto X^{n} \otimes x^{n},
\end{aligned}
$$

is an isomorphism of the associative algebras.

3.2. Construction of a class of $\mathbb{Z}^{d}$-graded $\mathbb{C}_{q}$-modules. We continue the notation in Section 3.1. Clearly, $\mathfrak{g l}_{N}$ is a $\Gamma$-graded Lie algebra with the gradation

$$
\mathfrak{g l}_{N}=\bigoplus_{\bar{n} \in \Gamma}\left(\mathfrak{g l}_{N}\right)_{\bar{n}},
$$

where $\left(\mathfrak{g l}_{N}\right)_{\bar{n}}=\operatorname{span}\left\{X^{n} \in \mathfrak{g l}_{N}\right\}$. In the following, by $\Gamma$-gradation of the Lie algebra $\mathfrak{g l}_{N}$ we shall always mean the above gradation.

A module $W$ over the Lie algebra $\mathfrak{g l}_{N}$ is called a $\Gamma$-graded $\mathfrak{g l}_{N}$-module if $W$ has a subspace decomposition

$$
W=\bigoplus_{\bar{n} \in \Gamma} W_{\bar{n}}
$$

such that $\left(\mathfrak{g l}_{N}\right)_{\bar{m}} W_{\bar{n}} \subset W_{\bar{m}+\bar{n}}$ for all $m, n \in \Gamma$. If $W$ is a $\Gamma$-graded $\mathfrak{g l}_{N}$-module with the above gradation, then for any $\bar{m} \in \Gamma, W=\bigoplus_{\bar{n} \in \Gamma} W_{\bar{n}+\bar{m}}$ is also a $\Gamma$-gradation of $W$.

For a $\Gamma$-graded module $W=\bigoplus_{\bar{n} \in \Gamma} W_{\bar{n}}$ over the Lie algebra $\mathfrak{g l}_{N}$, we can define $\mathbb{Z}^{d}$-graded module $\mathcal{V}(W)=\bigoplus_{n \in \mathbb{Z}^{d}} W_{\bar{n}}(n)$ over the Lie algebra $\mathbb{C}_{q}$ by the following actions

$$
t^{m} w_{\bar{n}}(n)=\left(X^{m} w_{\bar{n}}\right)(n+m), \quad w_{\bar{n}} \in W_{\bar{n}}, m, n \in \mathbb{Z}^{d} .
$$

Proposition 3.2. If $W=\bigoplus_{\bar{n} \in \Gamma} W_{\bar{n}}$ is a nontrivial $\Gamma$-graded-irreducible $\mathfrak{g l}_{N}$-module, then $\mathcal{V}(W)$ is a $\mathbb{Z}^{d}$-graded-irreducible module over the Lie algebra $\mathbb{C}_{q}$. 


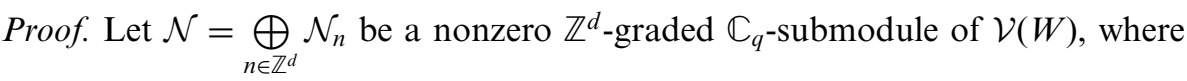
$\mathcal{N}_{n} \subset W_{\bar{n}}(n)$. Let

$$
\mathcal{N}_{\bar{n}}^{\prime}=\operatorname{span}\left\{w_{\bar{n}} \in W_{\bar{n}} \mid w_{\bar{n}}(n+r) \in \mathcal{N} \text { for each } r \in \operatorname{rad}(f)\right\},
$$

and $\mathcal{N}^{\prime}=\bigoplus_{\bar{n} \in \Gamma} \mathcal{N}_{\bar{n}}^{\prime}$. It is easy to check that $\mathcal{N}^{\prime}$ is a $\Gamma$-graded $\mathfrak{g l}_{N}$-submodule of $W$. Since $W$ is irreducible, then $\mathcal{N}^{\prime}=0$ or $W$. Fix $0 \neq w_{\bar{m}}(m) \in \mathcal{N}$. Since $W$ is nontrivial, there exists $X^{n^{\prime}} \in \mathfrak{g l}_{N}$ such that $X^{n^{\prime}} w_{\bar{m}} \neq 0$. From

$$
0 \neq t^{n^{\prime}+r} w_{\bar{m}}(m)=\left(X^{n^{\prime}} w_{\bar{m}}\right)\left(m+n^{\prime}+r\right) \in \mathcal{N}, \forall r \in \operatorname{rad}(f),
$$

we can see that $\mathcal{N}^{\prime} \neq 0$. So $\mathcal{N}^{\prime}=W$, hence $\mathcal{N}=\mathcal{V}(W)$.

COROLlary 3.3. If $W=\bigoplus_{\bar{n} \in \Gamma} W_{\bar{n}}$ is a nontrivial $\Gamma$-graded-irreducible $\mathfrak{g l}_{N}$-module, then the $\mathbb{C}_{q}$-module $\mathcal{L}(W)=W \otimes \mathbb{C}_{q}$ defined by

$$
t^{m} w(n)=\left(X^{m} w\right)(n+m), w \in W, m, n \in \mathbb{Z}^{d}
$$

is a $\mathbb{Z}^{d}$-graded-completely reducible $\mathbb{C}_{q}$-module and all irreducible submodules are isomorphic to $\mathcal{V}(W)$.

Proof. For $\bar{m} \in \Gamma$, we define $\mathbb{Z}^{d}$-graded $\mathbb{C}_{q}$-modules

$$
\mathcal{V}\left(W^{(\bar{m})}\right)=\bigoplus_{n \in \mathbb{Z}^{d}} W_{\bar{n}+\bar{m}}(n)
$$

It is easy to verify that $\mathcal{L}(W)=\bigoplus_{m \in \mathbb{Z}^{d}} \mathcal{V}\left(W^{(\bar{m})}\right)$, and $\mathcal{V}\left(W^{(\bar{m})}\right) \cong \mathcal{V}(W)$. Then we complete the proof.

3.3. Characterization of $\mathbb{Z}^{d}$-graded modules over the Lie algebra $\mathbb{C}_{q}$. Let $I$ be the idea of the associative algebra $Z\left(\mathbb{C}_{q}\right)$ spanned by $t^{r}-t^{s}$ where $r, s \in \operatorname{rad}(f)$. If the action of $Z\left(\mathbb{C}_{q}\right)$ on a $\mathbb{Z}^{d}$-graded module $M=\bigoplus_{n \in \mathbb{Z}^{d}} M_{n}$ over the Lie algebra $\mathbb{C}_{q}$ is associative, we define $M^{\prime}=I M$ which is a $\mathbb{C}_{q}$-submodule of $M$. Let $M^{F}=M / M^{\prime}$, which is a $\Gamma$-graded $\mathbb{C}_{q}$-module with homogeneous space $M_{\bar{n}}^{F}=\pi\left(\bigoplus_{r \in \operatorname{rad}(f)} M_{n+r}\right)$ for $n \in \mathbb{Z}^{d}$, where $\pi$ is the corresponding canonical map (also module homomorphism) from $M$ to $M^{F}$. The following result will be used several times later.

Lemma 3.4. Let $M=\bigoplus_{n \in \mathbb{Z}^{d}} M_{n}$ be a $\mathbb{Z}^{d}$-graded $\mathbb{C}_{q}$-module with all finitedimensional homogenous subspaces. If the action of $Z\left(\mathbb{C}_{q}\right)$ on $M$ is associative,

(a) the restriction $\pi: M_{n} \rightarrow M_{\bar{n}}^{F}$ is a vector space isomorphism for any $n \in \mathbb{Z}^{d}$;

(b) if $M$ is $\mathbb{Z}^{d}$-graded irreducible over $\mathbb{C}_{q}$ then $M^{F}$ is $\Gamma$-graded irreducible over $\mathbb{C}_{q}$.

Proof. (a) Let $M_{\bar{n}}=\bigoplus_{r \in \operatorname{rad}(f)} M_{n+r}$. Obviously $\pi$ is surjective. It suffices to check that $M_{n}$ and $M_{\bar{n}}^{F}$ have same dimension. Indeed, it is easy to check that $M_{\bar{n}} \cong Z^{k}=$ $Z \times Z \times \cdots \times Z$, as modules over the associative algebra $Z=Z\left(\mathbb{C}_{q}\right)$, where $k$ is the dimension of $M_{n}$. So $M_{\bar{n}}^{F} \cong Z^{k} / I Z^{k} \cong(Z / I)^{k} \cong \mathbb{C}^{k}$ as vector spaces, i.e., $M_{n}$ and $M_{\bar{n}}^{F}$ have same dimension.

(b) is clear. 
THEOREM 3.5. Let $M=\bigoplus_{n \in \mathbb{Z}^{d}} M_{n}$ be a $\mathbb{Z}^{d}$-graded module over the Lie algebra $\mathbb{C}_{q}$ with all finite-dimensional homogenous subspaces. If it satisfies that

$$
t^{0} v=v, t^{n+r} v=t^{n} t^{r} v, \forall n \in \mathbb{Z}^{d}, r \in \operatorname{rad}(f), v \in M .
$$

Then there exists a finite-dimensional $\Gamma$-graded $\mathfrak{g l}_{N}$-module $M^{F}$ such that $M$ is isomorphic to $\mathcal{V}\left(M^{F}\right)$.

Proof. From (3.1) we know that the action of $Z\left(\mathbb{C}_{q}\right)$ on $M$ is associative. For the above defined $M^{\prime}$ and $M^{F}$, from (3.1) we see that $\mathcal{I} M^{F}=0$. Then $M^{F}$ is a $\mathbb{C}_{q} / \mathcal{I}$-module, i.e., a $\mathfrak{g l}_{N}$-module whose module structure is given by $X^{m} \pi\left(v_{n}\right)=\pi\left(t^{m} v_{n}\right)$ for $m$, $n \in \mathbb{Z}^{d}$. Obviously, the identity matrix $E$ has identity action on $M^{F}$.

Since $M$ and $M^{\prime}$ have the obvious $\Gamma$-gradation, the sum $M^{F}=\sum_{\bar{n} \in \Gamma} M_{\bar{n}}^{F}$ is direct. So $M^{F}$ is a $\Gamma$-graded $\mathfrak{g l}_{N}$-module with gradation $M^{F}=\bigoplus_{\bar{n} \in \Gamma} M_{\bar{n}}^{F}$.

We define the following linear map:

$$
\varphi: M \rightarrow \mathcal{V}\left(M^{F}\right), \quad v_{n} \mapsto \pi\left(v_{n}\right)(n), \forall v_{n} \in M_{n}, n \in \mathbb{Z}^{d} .
$$

For any $m \in \mathbb{Z}^{d}, v_{n} \in M_{n}$ we have $\varphi\left(t^{m} v_{n}\right)=\pi\left(t^{m} v_{n}\right)(m+n)=\left(X^{m} \pi\left(v_{n}\right)\right)(m+n)=$ $t^{m}\left(\pi\left(v_{n}\right)(n)\right)=t^{m} \varphi\left(v_{n}\right)$, i.e., $\varphi$ is a $\mathbb{C}_{q}$-module homomorphism. By Lemma 3.4, $\varphi$ is bijective. So $\varphi$ is a $\mathbb{C}_{q}$-module isomorphism.

EXAMPLE 1 . Consider the adjoint $\mathbb{Z}^{d}$-graded $\mathbb{C}_{q}$-module $M=\mathbb{C}_{q}^{\prime}$. Let $W$ be the adjoint representation of $\mathfrak{g l}_{N}$ on $\mathfrak{s l}_{N}$. We can verify directly that $M \cong \mathcal{V}(W)$.

\section{4. $\mathfrak{g}$-modules with nonzero action of $Z\left(\mathbb{C}_{q}\right)$.}

4.1. Defining irreducible weight modules over $\mathfrak{g}$. In this section, we still assume that $q$ is of the form in Lemma 2.1. For any $\mathfrak{g l}_{d}$-module $V$, any $\Gamma$-graded $\mathfrak{g l}_{N}$-module $W=\bigoplus_{\bar{n} \in \Gamma} W_{\bar{n}}$, and any $\alpha \in \mathbb{C}^{d}$, let $\mathcal{V}^{\alpha}(V, W)=V \otimes \mathcal{V}(W), F^{\alpha}(V)=V \otimes \mathbb{C}_{q}$. Then $\mathcal{V}^{\alpha}(V, W)$ becomes a $\mathfrak{g}$-module if we define the following actions

(1) $t^{s}\left(v \otimes w_{\bar{n}}(n)\right)=v \otimes\left(X^{s} w_{\bar{n}}\right)(n+s)$;

(2) $D(u, r)\left(v \otimes w_{\bar{n}}(n)\right)=\left((u \mid n+\alpha) v+\left(r u^{T}\right) v\right) \otimes w_{\bar{n}}(n+r)$;

where $u \in \mathbb{C}^{d}, \quad v \in V, \quad w_{\bar{n}} \in W_{\bar{n}}$ and $r \in \operatorname{rad}(f), s \in \mathbb{Z}^{d}, X^{s} \in \mathfrak{g l}_{N}$ (as defined in Section 3.1).

And $F^{\alpha}(V)$ becomes a $\mathfrak{g}$-module if we define the following actions

(1) $t^{s} v(n)=\sigma(s, n) v(n+s)$;

(2) $D(u, r) v(n)=\left((u \mid n+\alpha) v+\left(r u^{T}\right) v\right)(n+r)$;

where $u \in \mathbb{C}^{d}, v \in V, r \in \operatorname{rad}(f), s, n \in \mathbb{Z}^{d}, v(n)=v \otimes t^{n}$.

We note that the action of $\mathbb{C}_{q}$ on $F^{\alpha}(V)$ is associative. Moreover, we can check that $F^{\alpha}(V) \cong \mathcal{V}^{\alpha}\left(V, M_{N}(\mathbb{C})\right)$, where $M_{N}(\mathbb{C})$ is the left regular module over $\mathfrak{g l}_{N}$, which is $\Gamma$-graded simple. Note that in the above definitions, we do not assume anything (irreducible, or weight modules) on the modules $V$ and $W$, but the modules $\mathcal{V}^{\alpha}(V, W)$, $F^{\alpha}(V)$ are weight modules over $\mathfrak{g}$.

Proposition 4.1. If $V$ is an irreducible $\mathfrak{g l}_{d}$-module and $W$ is a nontrivial $\Gamma$-gradedirreducible $\mathfrak{g l}_{N}$-module, then $\mathcal{V}^{\alpha}(V, W), F^{\alpha}(V)$ are irreducible weight $\mathfrak{g}$-modules.

Proof. We first prove that $\mathcal{V}^{\alpha}(V, W)$ is an irreducible weight $\mathfrak{g}$-module. Choose a basis $\left\{w_{i} \mid i \in I\right\}$ of $W_{\bar{n}}$. Suppose $M$ is a nonzero $\mathfrak{g}$-submodule of $\mathcal{V}^{\alpha}(V, W)$. Since $M$ 
is a weight $\mathfrak{g}$-module, it contains a nonzero element of the form $\sum_{j \in I^{\prime}} v_{j} \otimes w_{j}(n)$ for some $n \in \mathbb{Z}^{d}$ with nonzero $w_{j} \in W_{\bar{n}}, j \in I^{\prime}$, where $I^{\prime}$ is a finite subset of index set $I$. We can consider $W$ as a $\Gamma$-graded-irreducible $\mathbb{C}_{q}$-module since $\mathbb{C}_{q} / \mathcal{I}=\mathfrak{g l}_{N}$ (see Section 3.1). Fix an element $k \in I^{\prime}$. By Lemma 2.5, we see that $\operatorname{End}_{U\left(\mathbb{C}_{q}\right)}(W)_{0}=\mathbb{C}$. Then from Density theorem, there exists an $a \in U\left(\mathbb{C}_{q}\right)$ such that $a w_{j}=\delta_{j k} w_{k}, j \in I^{\prime}$. Then, we know that $v_{k} \otimes w_{k}\left(n^{\prime}\right) \in M$ for some $n^{\prime} \in \mathbb{Z}^{d}$. Considering the action of $\mathbb{C}_{q}$ on $v_{k} \otimes w_{k}\left(n^{\prime}\right) \in M$, we see that $v_{k} \otimes \mathcal{V}(W) \subset M$. Denote $V^{\prime}=\{v \in V \mid v \otimes \mathcal{V}(W) \subset M\}$. Clearly, $V^{\prime} \neq 0$, since $v_{k} \otimes \mathcal{V}(W) \subset M$. From the definition of $\mathcal{V}^{\alpha}(V, W)$, we can see that $V^{\prime}$ is a $\mathfrak{g l}_{d}$-submodule of $V$. Thus $V^{\prime}=V$. It follows that $M=\mathcal{V}^{\alpha}(V, W)$. Then $\mathcal{V}^{\alpha}(V, W)$ is irreducible.

The irreducibility of $F^{\alpha}(V)$ follows from the isomorphism $F^{\alpha}(V) \cong$ $\mathcal{V}^{\alpha}\left(V, M_{N}(\mathbb{C})\right)$.

If $V$ is a $\mathfrak{g l}_{d}$-module and $W$ is a $\Gamma$-graded $\mathfrak{g l}_{N}$-module, then $\mathcal{L}^{\alpha}(V, W)=$ $V \otimes W \otimes \mathbb{C}_{q}$ becomes a $\mathfrak{g}$-module via

(1) $t^{s}(v \otimes w(n))=v \otimes\left(X^{s} w\right)(n+s)$;

(2) $D(u, r)(v \otimes w(n))=\left((u \mid n+\alpha) v+\left(r u^{T}\right) v\right) \otimes w(n+r)$;

where $u \in \mathbb{C}^{d}, v \in V, w \in W$ and $r \in \operatorname{rad}(f), s \in \mathbb{Z}^{d}$.

COROLlary 4.2. If $V$ is an irreducible $\mathfrak{g l}_{d}$-module and $W$ is a nontrivial $\Gamma$-gradedirreducible $\mathfrak{g l}_{N}$-module, then the $\mathfrak{g}$-module $\mathcal{L}^{\alpha}(V, W)$ is a completely reducible $\mathfrak{g}$-module.

Proof. This easily follows from Corollary 3.3 and Proposition 4.1.

\subsection{Weight modules over $\mathfrak{g}$ with associative action of $Z\left(\mathbb{C}_{q}\right)$.}

Proposition 4.3. Suppose that the action of $Z\left(\mathbb{C}_{q}\right)$ is associative on an irreducible Harish Chandra $\mathfrak{g}$-module $M$. For a fixed nonzero $r \in \operatorname{rad}(f)$, we define linear operators on $M$ by

$$
g(m, k)=\sum_{i=0}^{k}(-1)^{i}\left(\begin{array}{l}
k \\
i
\end{array}\right) t^{m+i r} t^{-i r}, m \in \mathbb{Z}^{d}, k \in \mathbb{N} .
$$

Then there exists an $l \in \mathbb{N}$ such that

$$
g(m, l)(M)=0, \forall m \in \mathbb{Z}^{d} .
$$

Proof. For any $m \in \operatorname{rad}(f)$ (or $r=0)$ and $k \in \mathbb{N}$, we have

$$
g(m, k)(M)=\sum_{i=0}^{k}(-1)^{i}\left(\begin{array}{l}
k \\
i
\end{array}\right) t^{m}(M)=(1-1)^{k}\left(t^{m} M\right)=0
$$

i.e. (4.2) holds for any $m \in \operatorname{rad}(f)$. If $\mathbb{C}_{q}^{\prime} M=0,(4.2)$ is also clear. Thus we need only to consider (4.2) for $m \in \mathbb{Z}^{d} \backslash \operatorname{rad}(f)$ with $\mathbb{C}_{q}^{\prime} M \neq 0$ (which also implies $\mathbb{C}_{q}^{\prime} \neq 0$ ).

If there exists an $m \in \mathbb{Z}^{d} \backslash \operatorname{rad}(f)$ such that $t^{m} M=0$, since $\mathbb{C}_{q}^{\prime}$ is $\mathbb{Z}^{d}$-graded-simple, then $\mathbb{C}_{q}^{\prime} M=0$, contradicting the assumption. So

$$
t^{m} M \neq 0, \forall m \in \mathbb{Z}^{d} \backslash \operatorname{rad}(f) .
$$


For a fixed $m \in \mathbb{Z}^{d} \backslash \operatorname{rad}(f)$, since $M^{F}$ (see Section 3.3 for the definition) is finite dimensional, there exist integers $l_{m}^{\prime} \leq l_{m}$, and scalars $a_{i} \in \mathbb{C}, l_{m}^{\prime} \leq i \leq l_{m}$, with $a_{l_{m}^{\prime}} a_{l_{m}} \neq 0$, such that $\sum_{i=l_{m}^{\prime}}^{l_{m}} a_{i} t^{m+i r} t^{-i r}\left(M^{F}\right)=0$. By Lemma 3.4, $\sum_{i=l_{m}^{\prime}}^{l_{m}} a_{i} t^{m+i r} t^{-i r}(M)=0$. Then

$$
\sum_{i=l_{m}^{\prime}}^{l_{m}} a_{i} t^{m+i r} t^{-i r} t^{l_{m}^{\prime} r}(M)=\sum_{i=l_{m}^{\prime}}^{l_{m}} a_{i} t^{m+l_{m}^{\prime} r+\left(i-l_{m}^{\prime}\right) r} t^{-\left(i-l_{m}^{\prime}\right) r}(M)=0 .
$$

Replace $m$ by $m+l_{m}^{\prime} r$, we can assume $l_{m}^{\prime}=0, l_{m} \in \mathbb{Z}_{+}$. So we have

$$
\sum_{i=0}^{l_{m}} a_{i} t^{m+i r} t^{-i r}(M)=0
$$

for this $m \in \mathbb{Z}^{d} \backslash \operatorname{rad}(f)$. We assume that $l$ is the minimum $l_{m}$ for $m \in \mathbb{Z}^{d} \backslash \operatorname{rad}(f)$ that have the property (4.4) with $a_{0} a_{l_{m}} \neq 0$. We fix such an $m \in \mathbb{Z}^{d} \backslash \operatorname{rad}(f)$ satisfying $\sum_{i=0}^{l} a_{i} t^{m+i r} t^{-i r}(M)=0$ with $a_{0} a_{l} \neq 0$.

Then for any $n \in \mathbb{Z}^{d} \backslash \operatorname{rad}(f)$, we have $\left[t^{n}, \sum_{i=0}^{l} a_{i} t^{m+i r} t^{-i r}\right](M)=0$, i.e., $\sum_{i=0}^{l} a_{i}\left[t^{n}, t^{m+i r}\right] t^{-i r}(M)=0$. Since $\mathbb{C}_{q}^{\prime}$ is $\mathbb{Z}^{d}$-graded simple, we have

$$
\sum_{i=0}^{l} a_{i} t^{m^{\prime}+i r} t^{-i r}(M)=0, \forall m^{\prime} \in \mathbb{Z}^{d}
$$

By (4.3), we see that $l>0$.

For an $m \in \mathbb{Z}^{d} \backslash \operatorname{rad}(f)$, choose $u \in \mathbb{C}^{d} \backslash\{0\}$ such that $(u \mid m)=0$ and $(u \mid r) \neq 0$. Then

$$
\begin{aligned}
0 & =D(u,-r) \sum_{i=0}^{l} a_{i} t^{m+i r} t^{-i r}(M)=\sum_{i=0}^{l}\left[D(u,-r), a_{i} t^{m+i r} t^{-i r}\right](M) \\
& =\left(\sum_{i=0}^{l}(u \mid m+i r) a_{i} t^{m+(i-1) r} t^{-i r}-\sum_{i=0}^{l}(u \mid r) i a_{i} t^{m+i r} t^{-(i+1) r}\right)(M) \\
& =(u \mid r)\left(\sum_{i=1}^{l} i a_{i} t^{m+(i-1) r} t^{-i r}-\sum_{i=1}^{l} i a_{i} t^{m+i r} t^{-(i+1) r}\right)(M) \\
& =(u \mid r)\left(\sum_{i=1}^{l} i a_{i} t^{m+(i-1) r} t^{-i r}-\sum_{i=2}^{l+1}(i-1) a_{i-1} t^{m+(i-1) r} t^{-i r}\right)(M)
\end{aligned}
$$




$$
\begin{aligned}
=(u \mid & r)\left(a_{1} t^{m} t^{-r}-l a_{l} t^{m+l r} t^{-(l+1) r}\right. \\
& \left.+\sum_{i=2}^{l}\left(i a_{i}-(i-1) a_{i-1}\right) t^{m+(i-1) r} t^{-i r}\right)(M) \\
& =(u \mid r)\left(a_{1} t^{m}-l a_{l} t^{m+l r} t^{-l r}\right. \\
& \left.+\sum_{i=2}^{l}\left(i a_{i}-(i-1) a_{i-1}\right) t^{m+(i-1) r} t^{-(i-1) r}\right)\left(t^{-r} M\right) \\
& =(u \mid r)\left(a_{1} t^{m}-l a_{l} t^{m+l r} t^{-l r}+\sum_{i=1}^{l-1}\left((i+1) a_{i+1}-i a_{i}\right) t^{m+i r} t^{-i r}\right)(M)
\end{aligned}
$$

So

$$
\left(a_{1} t^{m}-l a_{l} t^{m+l r} t^{-l r}+\sum_{i=1}^{l-1}\left((i+1) a_{i+1}-i a_{i}\right) t^{m+i r} t^{-i r}\right)(M)=0 .
$$

Comparing (4.5) with (4.6), using the minimality of $l$ we obtain

$$
\frac{a_{1}}{a_{0}}=\frac{-l a_{l}}{a_{l}}=\frac{(i+1) a_{i+1}-i a_{i}}{a_{i}},
$$

i.e. $(i+1) a_{i+1}=(i-l) a_{i}, 0 \leq i \leq l-1$. Thus, we can assume $a_{i}=(-1)^{i}\left(\begin{array}{l}l \\ i\end{array}\right), 0 \leq i \leq l$. By (4.5) and the definition of $g(m, l),(4.2)$ follows.

THEOREM 4.4. Let $M$ be an irreducible Harish Chandra $\mathfrak{g}$-module. If the action of $Z\left(\mathbb{C}_{q}\right)$ on $M$ is associative, then there exist a finite-dimensional irreducible $\mathfrak{g l}_{d}$-module $V$, a finite-dimensional $\Gamma$-graded-irreducible $\mathfrak{g l}_{N}$-module $W$ with identity action of identity matrix $E$ in $\mathfrak{g l}_{N}$, and $\alpha \in \mathbb{C}^{d}$ such that $M \simeq \mathcal{V}^{\alpha}(V, W)$.

Proof. Since $M$ is an irreducible Harish Chandra $\mathfrak{g}$-module, there exists $\alpha \in \mathbb{C}^{d}$ such that $M=\bigoplus_{n \in \mathbb{Z}^{d}} M_{\alpha+n}$ is the weight space decomposition of $M$, where

$$
M_{\alpha+n}=\left\{v \in M \mid D(u, 0) v=(u \mid \alpha+n) v, \text { for all } u \in \mathbb{C}^{d}\right\} .
$$

This decomposition is also a $\mathbb{Z}^{d}$-gradation as $\mathbb{C}_{q}$-module.

If there exists a nonzero $v \in M$ such that $\mathbb{C}_{q}^{\prime} v=0$, let $B$ be the $\dot{\mathfrak{g}}$-submodule generated by $v$. Then $\mathbb{C}_{q}^{\prime} B=0$. Consequently, $M=B$ is an irreducible Harish Chandra $\dot{\mathfrak{g}}$-module with associative action of $Z\left(\mathbb{C}_{q}\right)$. In this case, by the isomorphism between $\dot{\mathfrak{g}}$ and $L$ in Lemma 2.3, using Eswara Rao's theorem in [10] or [5], we obtain our theorem with $W$ being one-dimensional $\mathfrak{g l}_{N}$-module. So, in the following we always assume that $\mathbb{C}_{q}^{\prime}$ does not annihilate any nonzero vectors in $M$.

Since $M$ is a nontrivial uniformly bounded $\mathbb{Z}^{d}$-graded module over the Lie algebra $\mathbb{C}_{q}$, it contains a nontrivial $\mathbb{Z}^{d}$-graded-irreducible $\mathbb{C}_{q}$-submodule $\mathcal{N}=$ $\bigoplus_{n \in \mathbb{Z}^{d}} \mathcal{N}_{n}$ with $\mathcal{N}_{n} \subset M_{\alpha+n}$. From Lemma 3.4, we know that $\mathcal{N}^{F}$ is a $\Gamma$-gradedirreducible $\mathbb{C}_{q}$-module (see Section 3.3 for $\mathcal{N}^{F}$ ).

We define a Lie subalgebra of the finite-dimensional Lie algebra $\operatorname{End}_{\mathbb{C}}\left(\mathcal{N}^{F}\right)$ by

$$
S=\operatorname{span}\left\{g(m, k) \mid m \in \mathbb{Z}^{d}, k \in \mathbb{N}\right\} .
$$


From

$$
\begin{aligned}
& {\left[g(m, k), g\left(m^{\prime}, k^{\prime}\right)\right]} \\
& =\left[\sum_{i=0}^{k}(-1)^{i}\left(\begin{array}{l}
k \\
i
\end{array}\right) t^{m+i r} t^{-i r}, \sum_{j=0}^{k^{\prime}}(-1)^{j}\left(\begin{array}{c}
k^{\prime} \\
j
\end{array}\right) t^{m^{\prime}+j r} t^{-j r}\right] \\
& =\sum_{i=0}^{k} \sum_{j=0}^{k^{\prime}}(-1)^{i+j}\left(\begin{array}{l}
k \\
i
\end{array}\right)\left(\begin{array}{l}
k^{\prime} \\
j
\end{array}\right)\left[t^{m+i r}, t^{m^{\prime}+j r}\right] t^{-(i+j) r} \\
& =\left(\sigma\left(m, m^{\prime}\right)-\sigma\left(m^{\prime}, m\right)\right) \sum_{i=0}^{k} \sum_{j=0}^{k^{\prime}}(-1)^{i+j}\left(\begin{array}{l}
k \\
i
\end{array}\right)\left(\begin{array}{l}
k^{\prime} \\
j
\end{array}\right) t^{m+n+(i+j) r} t^{-(i+j) r} \\
& =\left(\sigma\left(m, m^{\prime}\right)-\sigma\left(m^{\prime}, m\right)\right) \sum_{p=0}^{k+k^{\prime}}(-1)^{p} \sum_{i+j=p}\left(\begin{array}{l}
k \\
i
\end{array}\right)\left(\begin{array}{c}
k^{\prime} \\
j
\end{array}\right) t^{m+n+p r} t^{-p r} \\
& =\left(\sigma\left(m, m^{\prime}\right)-\sigma\left(m^{\prime}, m\right)\right) \sum_{p=0}^{k+k^{\prime}}(-1)^{p}\left(\begin{array}{c}
k+k^{\prime} \\
p
\end{array}\right) t^{m+n+p r} t^{-p r} \\
& =\left(\sigma\left(m, m^{\prime}\right)-\sigma\left(m^{\prime}, m\right)\right) g\left(m+m^{\prime}, k+k^{\prime}\right)
\end{aligned}
$$

and Proposition 4.3, we know that $S$ is a solvable Lie subalgebra of $\operatorname{End}_{\mathbb{C}}\left(\mathcal{N}^{F}\right)$. By Lie's theorem, there exists a nonzero vector $v^{*} \in \mathcal{N}^{F}$ such that $g(m, 1) v^{*}=\lambda(m) v^{*}$ with $\lambda(m) \in \mathbb{C}$ for any $m \in \mathbb{Z}^{d}$. In particular, $\lambda(r)=0$, for any $r \in \operatorname{rad}(f)$. Let $\mathcal{N}^{\prime}=U\left(\mathbb{C}_{q}\right) v^{*}$ which is not necessarily equal to $\mathcal{N}^{F}$ since $v^{*}$ may not be homogenous. It is easy to check that

$$
g(m, 1) t^{n} v^{*}=\lambda(m) t^{n} v^{*}+(\sigma(m, n)-\sigma(n, m)) \lambda(m+n) v^{*} .
$$

Since the $\mathbb{C}_{q}$-module $\mathcal{N}^{\prime}$ is generated by $v^{*}$, it follows from (4.7) that the matrices of all $g(m, 1) \in S$ ( relative to a suitable basis of $\mathcal{N}^{\prime}$ ) will be triangular, with $\lambda(m)$ the only diagonal entry. Since $\mathbb{C}_{q}^{\prime}$ is $\mathbb{Z}^{d}$-graded-simple, for any $m \in \mathbb{Z}^{d} \backslash \operatorname{rad}(f)$, there exist a $n \in \mathbb{Z}^{d} \backslash \operatorname{rad}(f)$ such that $\sigma(m, n)-\sigma(n, m) \neq 0$. Then

$$
g(m, 1)=(\sigma(m-n, n)-\sigma(n, m-n))^{-1}\left[g(m-n, 1), t^{n}\right]
$$

So $g(m, 1)$ has zero trace, this forces $\lambda(m)=0$. Therefore,

$$
g(m, 1) v^{*}=0, \text { in } \mathcal{N}^{F}, \forall m \in \mathbb{Z}^{d} .
$$

Since $g(m, 1)$ is a homogeneous operator of degree $m$, we can assume $v^{*}$ is a homogeneous element in the $\Gamma$-graded $\mathbb{C}_{q}$-module $\mathcal{N}^{F}$. However, $\mathcal{N}^{F}$ is a $\Gamma$-gradedirreducible $\mathbb{C}_{q}$-module, by $(4.7), g(m, 1) \mathcal{N}^{F}=0$, for any $m \in \mathbb{Z}^{d}$. By Lemma 3.4, $\pi(g(m, 1) \mathcal{N})=g(m, 1) \pi(\mathcal{N})=g(m, 1) \mathcal{N}^{F}=0$, consequently $g(m, 1) \mathcal{N}=0$, for any $m \in \mathbb{Z}^{d}$.

Denote

$$
U=\left\{v \in M \mid t^{0} v=v, t^{n+r} v=t^{n} t^{r} v \text {, for all } n \in \mathbb{Z}^{d}, r \in \operatorname{rad}(f)\right\}
$$


Obviously, $\mathcal{N} \subseteq U$. Then, it is easy to check that $U$ is a nonzero $\mathfrak{g}$-submodule of $M$ by the following calculation:

$$
\begin{aligned}
\left(t^{n+r}-t^{n} t^{r}\right) t^{s} v=(\sigma(n, s)- & \sigma(s, n))\left(t^{n+s+r}-t^{n+s} t^{r}\right) v+t^{s}\left(t^{n+r}-t^{n} t^{r}\right) v, \\
\left(t^{n+r}-t^{n} t^{r}\right) D\left(u, r^{\prime}\right) v= & -(u \mid n)\left(t^{n+r+r^{\prime}}-t^{n+r^{\prime}} t^{r}\right) v \\
& \quad-(u \mid r)\left(t^{n+r+r^{\prime}}-t^{n} t^{r+r^{\prime}}\right) v+D\left(u, r^{\prime}\right)\left(t^{n+r}-t^{n} t^{r}\right) v .
\end{aligned}
$$

By the irreducibility of $\mathfrak{g}$-module, we can see that $U=M$, i.e, $M$ satisfies the condition (3.1) in Theorem 3.5. Therefore, by Theorem 3.5, as $\mathbb{C}_{q}$-module, $M$ is isomorphic to $\mathcal{V}\left(W^{\prime}\right)$ for some $\Gamma$-graded $\mathfrak{g l}_{N}$-module $W^{\prime}=\bigoplus_{\bar{n} \in \Gamma} W_{\bar{n}}^{\prime}$ with identity action of the identity matrix $E$ subject to the following action:

$$
t^{m} w_{\bar{n}}(n)=\left(X^{m} w_{\bar{n}}\right)(n+m), w_{\bar{n}} \in W_{\bar{n}}^{\prime}, m, n \in \mathbb{Z}^{d},
$$

Note that $M_{\alpha+n}=W_{\bar{n}}^{\prime}(n)$.

For any $s \in \mathbb{Z}^{d}$, let $M_{\bar{s}}=\bigoplus_{r \in \operatorname{rad}(f)} M_{\alpha+s+r}=\bigoplus_{r \in \operatorname{rad}(f)} W_{\bar{s}}^{\prime}(s+r)$, which is a Harish Chandra $\dot{\mathfrak{g}}$-module with the associative action of $Z\left(\mathbb{C}_{q}\right)$. By the isomorphism between $\dot{\mathfrak{g}}$ and $L$, using Eswara Rao's Theorem [10], $M_{\overline{0}}$ has an irreducible $\dot{\mathfrak{g}}$-submodule of the form $V \otimes Z\left(\mathbb{C}_{q}\right)$ for some finite-dimensional irreducible $\mathfrak{g l}_{d}$-module $V$ (a subspace of $\left.W_{\overline{0}}^{\prime}\right)$ with the following action:

$$
D(u, r) v\left(r^{\prime}\right)=\left(u \mid \alpha+r^{\prime}\right) v\left(r^{\prime}+r\right)+\left(r u^{T}\right) v\left(r^{\prime}+r\right), \quad t^{r} v\left(r^{\prime}\right)=v\left(r^{\prime}+r\right)
$$

for all $r^{\prime}, r \in \operatorname{rad}(f), v \in V$.

We use $\mathcal{T}$ to denote the Lie subalgebra of $\operatorname{End}_{\mathbb{C}}(M)$ generated by operators $T(u, r)=t^{-r} D(u, r)-D(u, 0), r \in \operatorname{rad}(f), u \in \mathbb{C}^{d}$. It follows that, on $M$,

$$
D(u, r)=t^{r} T(u, r)+t^{r} D(u, 0), \quad \forall r \in \operatorname{rad}(f), u \in \mathbb{C}^{d} .
$$

Clearly $V$ (or more precisely, $V(0)$ ) is a $\mathcal{T}$-module (in fact, $\mathfrak{g l}_{d}$ is a quotient algebra of $\mathcal{T}$, see [10]). For any $s \in \mathbb{Z}^{d}, \quad r \in \operatorname{rad}(f), w_{\bar{n}}^{\prime} \in W_{\bar{n}}^{\prime}$, using (4.10) we have

$$
\begin{aligned}
T & (u, r) t^{s} w_{\bar{n}}^{\prime}(n) \\
& =\left(t^{-r} D(u, r)-D(u, 0)\right) t^{s} w_{\bar{n}}^{\prime}(n) \\
& \left.=\left[\left(t^{-r} D(u, r)-D(u, 0)\right)\right), t^{s}\right] w_{\bar{n}}^{\prime}(n)+t^{s}\left(t^{-r} D(u, r)-D(u, 0)\right) w_{\bar{n}}^{\prime}(n) \\
& =\left((u \mid s) t^{-r} t^{s+r}-(u \mid s) t^{s}\right) w_{\bar{n}}^{\prime}(n)+t^{s}\left(t^{-r} D(u, r)-D(u, 0)\right) w_{\bar{n}}^{\prime}(n) \\
& =t^{s}\left(t^{-r} D(u, r)-D(u, 0)\right) w_{\bar{n}}^{\prime}(n) \\
& =t^{s} T(u, r) w_{\bar{n}}^{\prime}(n) .
\end{aligned}
$$

Consequently, on $V$,

$$
T(u, r) X^{s}=X^{s} T(u, r), \quad \forall s \in \mathbb{Z}^{d}, r \in \operatorname{rad}(f) .
$$

Let $W=\bigoplus_{\bar{n} \in \Gamma} \operatorname{Hom}_{\mathcal{T}}\left(V, W_{\bar{n}}^{\prime}\right)$ which is obviously nonzero. Denote $W_{\bar{n}}=$ $\operatorname{Hom}_{\mathcal{T}}\left(V, W_{\bar{n}}^{\prime}\right)$. Define the action of $\mathfrak{g l}_{N}$ on $W$ :

$$
\left(X^{s} g_{\bar{n}}\right)(v)=X^{s} g_{\bar{n}}(v), \forall g_{\bar{n}} \in \operatorname{Hom}_{\mathcal{T}}\left(V, W_{\bar{n}}^{\prime}\right)
$$


From

$$
\begin{aligned}
\left(X^{s} g_{\bar{n}}\right)(T(u, r) v) & =X^{s} g_{\bar{n}}(T(u, r) v) \\
& =X^{s} T(u, r) g_{\bar{n}}(v) \\
& =T(u, r)\left(X^{s} g_{\bar{n}}\right)(v),(\text { by formula }(4.11))
\end{aligned}
$$

for each $v \in V, s \in \mathbb{Z}^{d}, g_{\bar{n}} \in W_{\bar{n}}$, we know that $W$ becomes a $\mathfrak{g l}_{N}$-module. From the fact that $X^{s} W_{\bar{n}} \subset W_{\bar{n}+s}$ we know that $W=\bigoplus_{\bar{n} \in \Gamma} W_{\bar{n}}$ is a $\Gamma$-graded $\mathfrak{g l}_{N}$-module.

We define the following linear map:

$$
\varphi: \mathcal{V}^{\alpha}(V, W) \rightarrow M, \quad v \otimes g_{\bar{n}}(n) \quad \mapsto g_{\bar{n}}(v)(n)
$$

Since $V \subset W_{\overline{0}}^{\prime}$, if we take $g_{\overline{0}}$ to be the imbedding map, we see that $\varphi\left(v \otimes g_{\overline{0}}(0)\right)=v(0)$ for any $v \in V$. So $\varphi \neq 0$. It is easy to check that

$$
\begin{aligned}
\varphi\left(t^{s}\left(v \otimes g_{\bar{n}}(n)\right)\right) & =\varphi\left(v \otimes\left(X^{s} g_{\bar{n}}\right)(n+s)\right) \\
& =X^{s} g_{\bar{n}}(v)(n+s)=t^{s}\left(g_{\bar{n}}(v)(n)\right) \\
& =t^{s} \varphi\left(v \otimes g_{\bar{n}}(n)\right)
\end{aligned}
$$

$$
\begin{aligned}
\varphi(D(u, r)(v & \left.\left.\otimes g_{\bar{n}}(n)\right)\right) \\
& =\varphi\left((u \mid n+\alpha) v \otimes g_{\bar{n}}(n+r)+r u^{T} v \otimes g_{\bar{n}}(n+r)\right) \\
& =(u \mid n+\alpha) g_{\bar{n}}(v)(n+r)+g_{\bar{n}}\left(r u^{T} v\right)(n+r)
\end{aligned}
$$

$$
\begin{aligned}
D(u, r) \varphi(v & \left.\otimes g_{\bar{n}}(n)\right) \\
& =D(u, r)\left(g_{\bar{n}}(v)(n)\right) \\
& =t^{r} T(u, r)\left(g_{\bar{n}}(v)(n)\right)+t^{r} D(u, 0) g_{\bar{n}}(v)(n) \\
& =t^{r} g_{\bar{n}}(T(u, r) v(n))+t^{r}(u \mid n+\alpha) g_{\bar{n}}(v)(n) \\
& =t^{r} g_{\bar{n}}\left(r u^{T} v\right)(n)+t^{r}(u \mid n+\alpha) g_{\bar{n}}(v)(n) \\
& =g_{\bar{n}}\left(r u^{T} v\right)(n+r)+(u \mid n+\alpha) g_{\bar{n}}(v)(n+r) .
\end{aligned}
$$

So, $\varphi$ is a $\mathfrak{g}$-module homomorphism. Since $M$ is an irreducible $\mathfrak{g}$-modules and $\varphi \neq 0, \varphi$ is surjective.

Let $\left\{v_{i} \mid i \in I\right\}$ be a basis for $V$. Suppose

$$
\varphi\left(\sum_{n \in \mathbb{Z}^{d}, i \in I} v_{i} \otimes g_{\bar{n}}^{(i)}(n)\right)=\sum_{n \in \mathbb{Z}^{d}, i \in I} g_{\bar{n}}^{(i)}\left(v_{i}\right)(n)=0,
$$

where $g_{\bar{n}}^{(i)} \in W_{\bar{n}}$ are 0 except for finitely many. Then $\sum_{i \in I} g_{\bar{n}}^{(i)}\left(v_{i}\right)(n)=0$ for each $n \in \mathbb{Z}^{d}$. Since $V$ is an irreducible $\mathfrak{g l}_{d}$-modules, we know that $\operatorname{End}_{\mathfrak{g l}_{d}}(V)=\mathbb{C}$. For any $k \in I$, by the Density Theorem, there exists an $a_{k} \in U(\mathcal{T})$, the universal enveloping algebra of $\mathcal{T}$, such that $a_{k} v_{i}=\delta_{i k} v_{k}$. Then

$$
a_{k} \sum_{i \in I} g_{\bar{n}}^{(i)}\left(v_{i}\right)=\sum_{i \in I} g_{\bar{n}}^{(i)}\left(a_{k} v_{i}\right)=g_{\bar{n}}^{(k)}\left(v_{k}\right)=0, \forall n \in \mathbb{Z}^{d}
$$


By the irreducibility of $\mathcal{T}$-module $V, g_{\bar{n}}^{(k)}(V)=0$, i.e., $g_{\bar{n}}^{(k)}=0$ for each $n \in \mathbb{Z}^{d}, k \in I$. Therefore $\varphi$ is injective. From the irreducibility of $\mathfrak{g}$-module $M$, we know that $W$ is a $\Gamma$-graded-irreducible $\mathfrak{g l}_{N}$-module. Now, we complete the proof of this theorem.

EXAMPLE 2. For any irreducible $\mathfrak{g l}_{d}$-module $V, G^{\alpha}(V)=V \otimes \mathbb{C}_{q}^{\prime}$ becomes a $\mathfrak{g}$-module by the following actions:

(1) $t^{r}\left(v \otimes t^{n}\right)=\sigma(r, n)\left(v \otimes t^{n+r}\right)$;

(2) $t^{s}\left(v \otimes t^{n}\right)=\sigma(s, n)(1-f(n, s))\left(v \otimes t^{n+s}\right)$;

(3) $D(u, r) v \otimes t^{n}=\sigma(r, n)\left((u \mid n+\alpha) v+\left(r u^{T}\right) v\right) \otimes t^{n+r}$;

where $u \in \mathbb{C}^{d}, v \in V, w \in W$ and $s, n \notin \operatorname{rad}(f), r \in \operatorname{rad}(f)$. From Theorem 4.1 in [23], $G^{\alpha}(V)$ is an irreducible Harish Chandra $\mathfrak{g}$-module. Let $W$ be the adjoint representation of $\mathfrak{s l}_{N}$. Using Example 1, we can see that $G^{\alpha}(V) \cong \mathcal{V}^{\alpha}(V, W)$.

In [10], Eswara Rao classified irreducible Harish Chandra modules over $\mathfrak{g}$ assuming that all $q_{i j}=1$ with the associative action of Laurent polynomials $\mathbb{C}_{q}$. He proved that any such module is a tensor module $F^{\alpha}(V)$. We will use Theorem 4.4 to give a quantum version of this result in the following corollary.

Corollary 4.5. Let $M$ be an irreducible Harish Chandra $\mathfrak{g}$-module. If the action of $\mathbb{C}_{q}$ on $M$ is associative, then there exist an irreducible finite-dimensional $\mathfrak{g l}_{d}$-module $V$, and $\alpha \in \mathbb{C}^{d}$ such that $M \simeq F^{\alpha}(V)$.

Proof. By Theorem 4.4, there exist a finite-dimensional irreducible $\mathfrak{g l}_{d}$-module $V^{\prime}$, a $\Gamma$-graded-irreducible $\mathfrak{g l}_{N}$-module $W=\bigoplus_{\bar{n} \in \Gamma} W_{\bar{n}}$ with identity action of identity matrix $E$ in $\mathfrak{g l}_{N}$ and $\alpha \in \mathbb{C}^{d}$ such that $M$ is isomorphic to $\mathcal{V}^{\alpha}\left(V^{\prime}, W\right)$ subject to the following actions:

(1) $t^{s}\left(v^{\prime} \otimes w_{\bar{n}}(n)\right)=v^{\prime} \otimes\left(X^{s} w_{\bar{n}}\right)(n+s)$;

(2) $D(u, r)\left(v^{\prime} \otimes w_{\bar{n}}(n)\right)=\left((u \mid n+\alpha) v^{\prime}+\left(r u^{T}\right) v\right) \otimes w_{\bar{n}}(n+r)$;

where $u \in \mathbb{C}^{d}, v^{\prime} \in V^{\prime}, w_{\bar{n}} \in W_{\bar{n}}$ and $r \in \operatorname{rad}(f), s \in \mathbb{Z}^{d}$. By (1) and associative action of $\mathbb{C}_{q}$, we can see that $\sigma(s, m) X^{s+m} w_{\bar{n}}=X^{s} X^{m} w_{\bar{n}}$, for each $s, m \in \mathbb{Z}^{d}$, so $W$ is $\Gamma$-gradedirreducible module over the associative algebra $\mathfrak{g l}_{N}$. Actually, $W$ is isomorphic to the left regular module $\mathfrak{g l}_{N}$. We may think $W=\mathfrak{g l}_{N}$, and $\mathcal{V}(W)=\sum_{n \in \mathbb{Z}^{d}} W_{\bar{n}}(n)=\mathbb{C}_{q}$ as a module over the Lie algebra $\mathbb{C}_{q}$ with the left associative multiplication action. We see that $M \simeq F^{\alpha}(V)$.

\subsection{Uniformly bounded $\mathfrak{g}$-modules with nonzero action of $Z\left(\mathbb{C}_{q}\right)$.}

THEOREM 4.6. Let $M$ be a uniformly bounded irreducible $\mathfrak{g}$-module with nonzero action of $Z\left(\mathbb{C}_{q}\right)$. Then there exist an irreducible finite-dimensional $\mathfrak{g l}_{d}$-module $V$, $a$ nontrivial finite-dimensional $\Gamma$-graded-irreducible $\mathfrak{g l}_{N}$-module $W$ (no restriction on the action of identity matrix $E$ in $\left.\mathfrak{g l}_{N}\right)$ and $\alpha \in \mathbb{C}^{d}$ such that $M$ is isomorphic to $\mathcal{V}^{\alpha}(V, W)$.

Proof. Let $U=\left\{v \in M \mid Z\left(\mathbb{C}_{q}\right) v=0\right\}$ which is obviously a $\mathfrak{g}$-submodule of $M$. Since $Z\left(\mathbb{C}_{q}\right) M \neq 0, U$ is a proper $\mathfrak{g}$-submodule of $M$. By the irreducibility of $\mathfrak{g}$-module $\mathrm{M}$, we see that $U=0$. Since $M$ is a uniformly bounded weight $\dot{\mathfrak{g}}$-module, it must contain a nontrivial irreducible weight $\dot{\mathfrak{g}}$-submodule $\mathcal{N}$. From $U=0$, we know that $Z\left(\mathbb{C}_{q}\right) \mathcal{N} \neq 0$. So $\mathcal{N}$ is a nontrivial irreducible weight $\dot{\mathfrak{g}}$-module with nonzero action of $Z\left(\mathbb{C}_{q}\right)$. By Theorem 2.4 and the fact that $\dot{\mathfrak{g}} \cong L$, we know that there exists a scalar $c \in \mathbb{C}^{*}$ such that

$$
t^{0} v=c v, c t^{r+r^{\prime}} v-t^{r} t^{r^{\prime}} v=0, \forall v \in N, \forall r, r^{\prime} \in \operatorname{rad}(f) .
$$


So the subspace

$$
U^{\prime}=\left\{v \in M \mid t^{0} v=c v, c t^{r+r^{\prime}} v-t^{r} t^{r^{\prime}} v=0, \text { for all } r, r^{\prime} \in \operatorname{rad}(f)\right\}
$$

of $M$ is nonzero. It is easy to check that $U^{\prime}$ is a $\mathfrak{g}$-submodule of $M$. By the irreducibility of $\mathfrak{g}$-module $M$, we can see that $U^{\prime}=M$. Let $t_{0}^{r}=t^{r} / c$ for each $r \in \operatorname{rad}(f)$. Then we have

$$
\left[t_{0}^{r}, t_{0}^{r^{\prime}}\right]=0,\left[D(u, r), t_{0}^{r^{\prime}}\right]=\left(u \mid r^{\prime}\right) t_{0}^{r+r^{\prime}}, \forall r, r^{\prime} \in \operatorname{rad}(f)
$$

By $U^{\prime}=M$, we can see that

$$
t_{0}^{0} v=v, t_{0}^{r+r^{\prime}} v=t_{0}^{r} r_{0}^{r^{\prime}} v, \forall v \in M, \forall r, r^{\prime} \in \operatorname{rad}(f)
$$

Then by Theorem 4.4, there exist an irreducible finite-dimensional $\mathfrak{g l}_{d}$-module $V$, a nontrivial $\Gamma$-graded-irreducible $\mathfrak{g l}_{N}$-module $W$ with scalar multiplication by $c$ of identity matrix $E$ in $\mathfrak{g l}_{N}$ and $\alpha \in \mathbb{C}^{d}$ such that $M$ is isomorphic to $\mathcal{V}^{\alpha}(V, W)$.

It is reasonable to expect that any irreducible uniformly bounded module over $\mathfrak{g}$ is of the form $\mathcal{V}^{\alpha}(V, W)$.

ACKNOWLEDGEMENT. K.Z. was partially supported by NSERC and the NSF of China (grant 10871192).

\section{REFERENCES}

1. B. Allison, S. Azam, S. Berman, Y. Gao and A. Pianzola, Extended affine Lie algebras and their root systems, Memoirs of the American Mathematical Society 126, vol. 605 (AMS, Providence, RI, 1997).

2. B. Allison, S. Berman, J. Faulkner and A. Pianzola, Realization of graded-simple algebras as loop algebras, Forum Mathematicum 20 (2008), 395-432.

3. S. Berman, Y. Gao and Y. S. Krylyuk, Quantum tori and the structure of elliptic quasisimple Lie algebras, J. Funct. Anal. 135 (1996), 339-389.

4. Y. Billig, A category of modules for the full toroidal Lie algebra, Int. Math. Res. Not. (2006), Art. ID 68395, 46.

5. Y. Billig, Jet modules, Canad. J. Math. 59(4) (2007), 712-729.

6. Y. Billig and M. Lau, Thin coverings of modules, J. Algebra 316 (2007), 147-173.

7. Y. Billig, A. Molev and R. Zhang, Differential equations in vertex algebras and simple modules for the Lie algebra of vector fields on a torus, Adv. Math. 218(6) (2008), 1972-2004.

8. Y. Billig and K. Zhao, Weight modules over exp-polynomial Lie algebras, J. Pure Appl. Algebra, 191(1-2) (2004), 23-42.

9. S. Eswara Rao, Irreducible representations of the Lie-algebra of the diffeomorphisms of a $d$-dimensional torus, J. Algebra 182(2) (1996), 401-421.

10. S. Eswara Rao, Partial classification of modules for Lie- algebra of diffeomorphisms of d-dimensional torus, J. Math. Phys., 45(8) (2004), 3322-3333.

11. S. Eswara Rao, Irreducible representations for Toroidal Lie algebras, J. Pure Appl. Algebra 202 (2005), 102-117.

12. S. Eswara Rao and C. Jiang, Classification of irreducible integrable representations for the full toroidal Lie algebras, J. Pure Appl. Algebra 200(1-2) (2005), 71-85.

13. J. M. Gracia-Bondia, J. C. Vasilly and H. Figueroa, Elements of non- commutative geometry (Birkhauser Advanced Texts, Birkhauser Verlag, Basel, Switzerland, 2001).

14. X. Guo, G. Liu and K. Zhao, Irreducible Harish Chandra modules over extended Witt algebras, Ark. Mat. doi:10.1007/s11512-012-0173-9. 
15. X. Guo and K. Zhao, Irreducible weight modules over Witt algebras, Proc. Amer. Math. Soc. 139 (2011), 2367-2373.

16. N. Jacbson, Basic algebra, II (W. H. Freeman and Company, San Francisco, CA, 1980).

17. V. Kac and A. Raina, Bombay lectures on highest weight representations of infinite dimensional Lie algebras (World Sci., Singapore, 1987).

18. T. A. Larsson, Multi-dimensional Virasoro algebra, Phys. Lett. B 231 (1989), 94-96.

19. T. A. Larsson, Central and non-central extensions of multi-graded Lie algebras, J. Phys. A 25 (1992), 1177-1184.

20. T. A. Larsson, Conformal fields: A class of representations of Vect (N), Int. J. Mod. Phys. A 7 (1992), 6493-6508.

21. T. A. Larsson, Lowest energy representations of non-centrally extended diffeomorphism algebras, Commun. Math. Phys. 201(1999), 461-470.

22. T. A. Larsson, Extended diffeomorphism algebras and trajectories in jet space, Commun. Math. Phys. 214 (2000), 469-491.

23. G. Liu and K. Zhao, Irreducible modules over the derivation algebras of rational quantum tori, J. Algebra 340 (2011), 28-34 .

24. $\mathrm{R}$. $\mathrm{Lu}$ and $\mathrm{K}$. Zhao, Classification of irreducible weight modules over higher rank Virasoro algebras, Adv. Math. 201(2) (2006), 630-656.

25. O. Mathieu, Classification of Harish Chandra modules over the Virasoro Lie algebra, Invent. Math. 107(2) (1992), 225-234.

26. O. Mathieu, Classification of irreducible weight modules, Ann. Inst. Fourier (Grenoble) 50(2) (2000), 537-592.

27. V. Mazorchuk, On simple mixed modules over the Virasoro algebra, Mat. Stud. 22(2) (2004), 121-128.

28. V. Mazorchuk, Lectures on $\mathfrak{s l}_{2}(\mathbb{C})$-modules (Imperial College Press, 2010).

29. V. Marzuchuk and K. Zhao, Supports of weight modules over Witt algebras, Proc. $R$. Soc. Edinburgh A 141(1) (2011), 155-170.

30. K. Neeb, On the classification of rational quantum tori and the structure of their automorphism groups, Canad. Math. Bull. 51(2) (2008), 261-282.

31. E. Ramos, C. H. Sah and R. E. Shrock, Algebras of diffeomorphisms of the N-torus, J. Math. Phys. 31(8) (1990), 1805-1816.

32. G. Shen, Graded modules of graded Lie algebras of Cartan type. I. Mixed products of modules, Sci. Sinica Ser. A 29(6) (1986), 570-581.

33. K. Zhao, Weight modules over generalized Witt algebras with 1-dimensional weight spaces, Forum Math. 16(5) (2004), 725-748.

34. K. Zhao, The q-Virasoro-like algebra, J. Algebra (1997), 506-512. 